\title{
Tafenoquine treatment of Plasmodium vivax malaria: suggestive evidence that CYP2D6 reduced metabolism is not associated with relapse in the Phase $2 \mathrm{~b}$ DETECTIVE trial
}

\author{
Pamela L. St Jean ${ }^{1 *} \mathbb{D}$, Zhengyu Xue ${ }^{1}$, Nick Carter ${ }^{2}$, Gavin C. K. W. Koh², Stephan Duparc ${ }^{3}$, Maxine Taylor ${ }^{4}$, \\ Claire Beaumont ${ }^{4}$, Alejandro Llanos-Cuentas ${ }^{5}$, Ronnatrai Rueangweerayut ${ }^{6}$, Srivicha Krudsood ${ }^{7}$, \\ Justin A. Green ${ }^{2}$ and Justin P. Rubio ${ }^{8}$
}

\begin{abstract}
Background: Tafenoquine (TQ) and primaquine (PQ) are 8-aminoquinolines (8-AQ) with anti-hypnozoite activity against vivax malaria. PQ is the only FDA-approved medicine for preventing relapsing Plasmodium vivax infection and TQ is currently in phase 3 clinical trials for the same indication. Recent studies have provided evidence that cytochrome P450 (CYP) metabolism via CYP2D6 plays a role in PQ efficacy against $P$. vivax and have suggested that this effect may extend to other 8-AQs, including TQ. Here, a retrospective pharmacogenetic (PGX) investigation was performed to assess the impact of CYP2D6 metabolism on TQ and PQ efficacy in the treatment of P. vivax in the DETECTIVE study (TAF112582), a recently completed, randomized, phase $2 \mathrm{~b}$ dose-ranging clinical trial. The impact of CYP2D6 on TQ pharmacokinetics (PK) was also investigated in TAF112582 TQ-treated subjects and in vitro CYP metabolism of TQ was explored. A limitation of the current study is that TAF112582 was not designed to be well powered for PGx, thus our findings are based on TQ or PQ efficacy in CYP2D6 intermediate metabolizers (IM), as there were insufficient poor metabolizers (PM) to draw any conclusion on the impact of the PM phenotype on efficacy.
\end{abstract}

Methods: The impact of genetically-predicted CYP2D6 reduced metabolism on relapse-free efficacy six months post-dosing of TQ or PQ, both administered in conjunction with chloroquine (CQ), was assessed using exact statistical methods in 198 P. vivax-infected study participants comparing IM to extensive metabolizers (EM). The influence of CYP2D6 metabolizer phenotypes on TQ PK was assessed comparing median TQ area under the curve (AUC). In vitro metabolism of TQ was investigated using recombinant, over-expressed human CYP enzymes and human hepatocytes. Metabolite identification experiments were performed using liquid chromatography-mass spectrometry.

Results: Reduction of CYP2D6 activity was not associated with an increase in relapse-rate in TQ-treated subjects $(p=0.57)$. In contrast, and in accordance with recent literature, CYP2D6 IMs were more common $(p=0.05)$ in PQtreated subjects who relapsed (50 \%) than in subjects who remained relapse-free (17\%). Further, CYP2D6 metabolizer phenotypes had no significant effect on TQ AUC, and only minimal metabolism of TQ could be detected in hepatic in vitro systems.

Conclusion: Together, these data provide preliminary evidence that in CYP2D6 IMs, TQ efficacy in P. vivax-infected individuals is not diminished to the same extent as PQ. As there were no PMs in either the TQ or PQ treatment arms of TAF112582, no conclusions could be drawn on potential differences in PMs. These findings suggest that differential

*Correspondence: pam.stjean@parexel.com

1 PAREXEL International, 2520 Meridian Parkway, Durham, NC 27713, USA

Full list of author information is available at the end of the article 
effects of CYP2D6 metabolism on TQ and PQ efficacy could be a differentiation factor between these 8-AQs, but results remain to be confirmed prospectively in the ongoing phase 3 studies.

Keywords: Plasmodium vivax malaria, Tafenoquine, Primaquine, CYP2D6, Efficacy, Pharmacogenetics, Pharmacokinetics

\section{Background}

Globally, it is estimated that $132-391$ million Plasmodium vivax clinical infections occur each year [1]. In order to treat and potentially eradicate relapsing forms of malaria, such as $P$. vivax, drugs with anti-hypnozoite activity are required to kill the latent hypnozoite liverstage of the parasite. Currently, primaquine (PQ) is the only drug approved by the US Food and Drug Administration (FDA) for this indication since schizonticidal drugs such as chloroquine (CQ) do not have anti-relapse efficacy. PQ is a member of the 8-aminoquinoline (8-AQ) drug class, which also includes tafenoquine (TQ). TQ is a synthetic derivative of $P Q$ and is currently in phase 3 clinical development after showing a good efficacy and tolerability profile in a recent phase $2 \mathrm{~b}$ dose-ranging study [2].

Recent research has highlighted a potential pharmacogenetic (PGx) effect on the efficacy of PQ in individuals who are naturally deficient in cytochrome $\mathrm{P} 450$ 2D6 (CYP2D6) activity. In both mice and humans, this research has provided consistent evidence that metabolic activation of PQ by CYP2D6 is required for anti-hypnozoite activity, possibly via a toxic metabolite [3-7]. A further study in mice suggested that this CYP2D6 liability may extend to other members of the 8-AQ drug class, including TQ [8]. Most recently, Vuong et al. [9] reported higher TQ exposure [area under the curve (AUC)] in CYP2D knock-out mice compared to wild type and suggested CYP2D6 could potentially affect TQ pharmacokinetics (PK) in humans.

CYP2D6 is a highly polymorphic drug-metabolizing enzyme involved in the biotransformation of numerous medicines and it is well known that genetic polymorphisms in the CYP2D6 gene influence exposure to many drugs and/or active metabolites and responses $[10,11]$. Metabolizer phenotype can be predicted for CYP2D6 alleles, although underlying metabolic differences between individuals with the same CYP2D6 genotype can make phenotype inference challenging [10-13]. If, as proposed by Marcsisin et al. [8], the CYP2D6 liability observed for PQ extends to other members of the 8-AQ drug class, the high degree of genetic polymorphism and considerable variability in the distribution of functional alleles across the world, would pose major obstacles to the development of pharmacogenetically guided treatment strategies.
The aim of the current study was to determine whether clinical anti-relapse efficacy of TQ and PQ, as well as TQ PK, are impacted by reduced CYP2D6 activity using a retrospective PGx assessment in subjects from a randomized clinical trial. A complimentary aim was to elucidate potential metabolic effects of CYP enzymes on TQ metabolism in vitro.

\section{Methods}

\section{Pharmacogenetic study subjects}

Participants' samples were from Part 1 of the seamless $\mathrm{Ph} 2 \mathrm{~b} / 3$ TAF112582 study, a multi-centred, double-blind, randomized, parallel-group, placebo-controlled study to evaluate the efficacy, safety and tolerability of TQ in subjects infected with $P$. vivax [2]. TAF112582 Part 1 consisted of six treatment arms: CQ (600 mg days 1 and 2, $300 \mathrm{mg}$ day 3) plus TQ and PQ placebos; CQ (doses as above) combined with single dose TQ 50, 100, 300 or $600 \mathrm{mg}$ plus PQ placebo; or CQ (doses as above) combined with PQ $15 \mathrm{mg}$ daily for 14 days plus TQ placebo. Protocol approval was obtained from each site's ethics committee or institutional review board and prospective written informed consent was obtained for all subjects involved in this PGx study, which was funded by GlaxoSmithKline (GSK) and the Medicines for Malaria Venture (MMV).

\section{Tafenoquine pharmacokinetics data}

A population PK model was developed to characterize systemic TQ concentrations in TAF112582 Part 1 subjects treated with TQ. Model-predicted individual post hoc clearance estimates were utilized to generate the individual exposure (AUC) values for the analyses [14].

\section{CYP2D6 genotyping and phenotype inference}

Venous blood was collected into an EDTA vacutainer for each of the subjects who consented to PGx research. Genomic DNA was extracted from peripheral blood using the Gentra Puregene kit on the Autopure LS (Qiagen, Valencia, CA, USA) by Quest Diagnostics (Valencia, CA, USA or Heston, UK). CYP2D6 genotyping was performed by BioProcessing Solutions (Piscataway, NJ, USA) using the Affymetrix ${ }^{\circledR}$ DMET-Plus array (Santa Clara, CA, USA). All genotype calling and quality control was performed in accordance with the manufacturers' protocols. 
Genetically predicted CYP2D6 metabolizer status was determined from this array. The translation of genotype information into metabolizer phenotype is challenging given the range of activity possible for an allele [12] and many classification schemes have been proposed or used in practice $[5,7,12]$. Here, subjects were classified as (1) poor metabolizers (PM) if they carried two 'null' (no enzymatic function) alleles; (2) intermediate metabolizers (IM) if they carried one null and one functionally deficient allele, two deficient alleles, or one null allele and one normal (wildtype function) allele; and, (3) extensive metabolizers (EM) if they carried two normal alleles or one normal allele and one deficient allele. In addition, the CYP2D6 'activity score' (AS) [12], which translates genotype into a qualitative measure of enzyme activity, was explored in this study. Briefly, for each study subject, AS was determined by summing the per-allele scores; a null allele having a score of 0 , a deficient allele a score of 0.5 and a normal allele a score of 1 . Thus, PMs could have an AS of 0 , IMs an AS of 0.5 or 1 and EMs an AS of 1.5 or 2.0.

\section{Statistical analysis}

TAF112582 showed poor efficacy for TQ50 and TQ100 mg treatment arms, while higher dose treatment arms, TQ300 and TQ600 mg, showed good efficacy [2]. Therefore, to facilitate interpretation of results, the TQ low dose (50 and $100 \mathrm{mg}$ ) and high dose (300 and $600 \mathrm{mg}$ ) treatment arms were combined. An exact logistic regression model adjusting for country of origin was used to assess the effect of inferred CYP2D6 metabolizer phenotype or AS on clinical outcome within treatment arm. Because only 1 PM was identified in the clinical study, only subjects with IM or EM phenotypes were included in the analysis. Given published data indicating an effect of CYP2D6 metabolism on PQ efficacy [3-7], a one-sided test was applied to test the one-sided alternative hypothesis that reduced metabolism increased the risk of relapse due to $P$. vivax infection 6 months postdosing. For the PK assessment, a post hoc analysis using a non-parametric method to compare median TQ AUC between IM and EM subjects was conducted separately in the TQ low dose (50 or $100 \mathrm{mg}$ ) and TQ high dose (300 or $600 \mathrm{mg}$ ) groups. All analyses were conducted using SAS/STAT (SAS Systemv9.2, SAS Institute Inc, Cary, NC, USA).

\section{Chemicals in isoenzyme and hepatocyte experiments}

Tafenoquine was supplied by Chemical Development, GlaxoSmithKline Research and Development Ltd. (Stevenage, UK). Glucose-6-phosphate (G6P), glucose6-phosphate dehydrogenase (G6PD) and $\beta$-nicotinamide adenine dinucleotide phosphate (NADP) were obtained from Sigma Chemical Company (St Louis, USA). All other commercially obtained chemicals and solvents were of high-performance liquid chromatography (HPLC) or analytical grade.

\section{Isoenzymes}

Supersomes ${ }^{\mathrm{TM}}$, containing individually over-expressed human CYP enzymes, derived from baculovirus-infected insect cells, and control Supersomes ${ }^{\mathrm{TM}}$ (lacking any native human CYP activity) were obtained from BD Biosciences, Woburn, MA, USA. The enzymes used in this study were: CYP1A2, CYP2C8, CYP2C9, CYP2C19, CYP2D6, CYP3A4 (Lot\# 72600, 76739, 73030, 71916, 73755 , and 70741, respectively). Supersomes ${ }^{\mathrm{TM}}$ expressing CYP2C8, CYP2C9, CYP2C19, and CYP3A4 co-expressed CYP reductase and cytochrome $b_{5}$, while Supersomes ${ }^{\text {TM }}$ expressing CYP1A2 and CYP2D6 co-expressed CYP reductase only. TQ was incubated at 5 and $10 \mu \mathrm{M}$ with Supersomes $^{\mathrm{TM}}$ (BD Gentest, Woburn, MA, USA), overexpressing individual cytochrome P450 enzymes 1A2, 2C8, $2 \mathrm{C} 9,2 \mathrm{C} 19,2 \mathrm{D} 6$, or $3 \mathrm{~A} 4$ at $300 \mathrm{pmol} / \mathrm{ml} \mathrm{P} 450$, in phosphate buffer $(50 \mathrm{mM}, \mathrm{pH} 7.4)$ at $37^{\circ} \mathrm{C}$ in a shaking water bath. Following an equilibration period of $5 \mathrm{~min}$ at $37^{\circ} \mathrm{C}$, reactions were started by the addition of pre-warmed cofactor solution [a NADPH regenerating system containing $1.7 \mathrm{mg}$ of NADP, $7.8 \mathrm{mg}$ of G6P, and six units of G6PD per $\mathrm{ml}$ of $2 \%(\mathrm{w} / \mathrm{v})$ sodium hydrogen carbonate]. Incubations were conducted under conditions which limited the exposure of samples to light. All incubations were terminated after 120 min by addition of a volume of acetonitrile equal to the incubation volume. Control incubations were also performed in the absence of Supersomes $^{\mathrm{TM}}$, in the absence of cofactor and in the absence of TQ. Samples were centrifuged (Eppendorf, Hauppauge, NY, USA; approximately $13,000 \mathrm{~g}, 3 \mathrm{~min}$, room temperature), and supernatants removed for analysis by ultra performance liquid chromatography-mass spectrometry (UPLC-MS).

\section{Human hepatocyte relay method}

The human hepatocyte relay method used was similar to that previously reported by Di et al. [15]. Pooled cryopreserved human hepatocytes from 26 donors were obtained from Life Technologies (MA, USA). Following thawing (as per supplier guidance), hepatocytes were resuspended in Williams' E medium (Sigma) supplemented with cell maintenance pack (Life Technologies). The cells were counted using the trypan blue exclusion method and 12-well hepatocyte plates containing 1 million cells/ $\mathrm{ml}$ were spiked with TQ at a final concentration of 5 and $10 \mu \mathrm{M}$ in a final incubation volume of $0.50 \mathrm{ml}$. The plates were incubated at $37{ }^{\circ} \mathrm{C}$ with $95 \%$ oxygen $/ 5 \%$ carbon dioxide, $75 \%$ relative humidity for $24 \mathrm{~h}$ on an orbital 
shaker in an incubator. At $24 \mathrm{~h}$ the hepatocyte suspension in the incubation plate was centrifuged $(13,000 \mathrm{~g}, 3 \mathrm{~min}$, room temperature); a total of $600 \mu \mathrm{l}$ of the supernatant was transferred to a clean 12 -well plate and stored at $-80{ }^{\circ} \mathrm{C}$ until the next relay experiment. For the next relay experiment, the supernatant plates were pre-warmed to $37^{\circ} \mathrm{C}$ for approximately $20 \mathrm{~min}$, and further hepatocytes (prepared as described previously) were added to the samples to yield a final cell density of 1 million cells $/ \mathrm{ml}$. The plates were incubated at $37^{\circ} \mathrm{C}$ for $24 \mathrm{~h}$ and processed as described earlier. Six relays were undertaken to give a total incubation time of 6 days and samples were protected from light wherever possible. A drug-only control plate was run under the same conditions to investigate any non-metabolic drug breakdown. At the end of the six relay method period the entire hepatocyte suspension was collected and centrifuged (Eppendorf, Hauppauge, NY, USA) at 13,000 $g$ for $3 \mathrm{~min}$ at room temperature, and transferred to vials prior to UPLC-MS analysis.

\section{Tafenoquine analysis and structural identification of drug related components}

Analyses of reaction phenotyping samples for TQ-related components were carried out by UPLC-MS using a Waters Acquity UPLC connected to a Waters Synapt G2S mass spectrometer using an Acquity UPLC BEH C18 column $(100 \times 2.1 \mathrm{~mm}, 1.7 \mu \mathrm{m}$; Waters Corporation, Milford, MA, USA). The mobile phase consisted of $50 \mathrm{mM}$ ammonium acetate (native $\mathrm{pH}$; solvent $\mathrm{A}$ ) and acetonitrile (solvent B) at a flow rate of $0.3 \mathrm{ml} / \mathrm{min}$. A gradient was used, starting at $5 \% \mathrm{~B}$ then linear phase to $95 \% \mathrm{~B}$ by $12 \mathrm{~min}$, held for $1 \mathrm{~min}$ before returning to the initial conditions by $13.5 \mathrm{~min}$ with these conditions being maintained for an additional $1.5 \mathrm{~min}$ before subsequent injections. TQ $\left([\mathrm{M}+\mathrm{H}]^{+}\right)$was detected at $m / z 464$ using positive ion electrospray ionization. Drug-related components were identified based on charged molecular ions, mass accuracy and their collision-induced dissociation fragmentation. Authentic standards, when available, were used to compare chromatographic retention times and fragmentation patterns. Supporting data from previous studies were also used in the assignment of structures (unpublished data on file within DMPK (Drug Metabolism and Pharmacokinetics) Department, GSK).

\section{Results}

Among 329 subjects who comprised the intent-to-treat (ITT) population in TAF112582, 237 (72 \%) provided written informed consent and a blood sample for PGx research; this included 136/136 (100\%) of subjects from Peru, 98/99 (99 \%) from Thailand, 3/57 (5\%) from India and 0/37 (0\%) from Brazil. Genetic data on 224 of the 237 (95\%) subjects passed standard manufacturer data quality control. Among these 224 subjects, 20 subjects were excluded from analysis as they were either lost to follow-up or treated with non-study medications with potential anti-malarial properties. In a further five subjects, metabolizer phenotype could not be unambiguously determined from CYP2D6 genotypes, leaving a PGx analysis sample of 199 subjects. The distribution of these 199 subjects by treatment, country of origin and clinical outcome is shown in Table 1. For the post hoc PK analysis, data were available on 128 of the 134 subjects treated with TQ that were included in the PGx analysis.

One of the 199 subjects evaluated was classified as PM (0.5 \%), 38 were classified as IM (19.1\%) and 160 were classified as EM (80.4\%) (Table 2). All three subjects from India were EMs. From Peru, there was one PM (0.9\%), 12 IMs (10.5\%) and 101 EMs (88.6\%), while from Thailand, there were 26 IMs $(31.7 \%)$ and 56 EMs (68.3\%). There was a statistically significant difference $(\mathrm{p}<0.001)$ in the frequency of the CYP2D6 IM phenotype between Peru ( $\mathrm{n}=12 / 114,10.5 \%$ ) and Thailand ( $\mathrm{n}=26 / 82,31.7 \%$ ) (see Additional file 1), but not between the four treatment arms (TQ high dose $+\mathrm{CQ}$, TQ low dose $+\mathrm{CQ}$, $\mathrm{PQ}+\mathrm{CQ}$, CQ only; $\mathrm{p}=0.09$ ) (see Additional file 2).

Table 1 Clinical outcome and country by treatment in 199 subjects involved in the CYP2D6 PGx analysis

\begin{tabular}{|c|c|c|c|c|c|c|}
\hline & \multicolumn{4}{|c|}{ TQ plus CQ ( $n=134$, total) } & \multirow{3}{*}{$\begin{array}{l}\text { PQ plus CQ } \\
(n=31)\end{array}$} & \multirow{3}{*}{$\begin{array}{l}\text { CQ plus placebo } \\
(n=34)\end{array}$} \\
\hline & $50 \mathrm{mg}(\mathrm{n}=34)$ & $\begin{array}{l}100 \mathrm{mg} \\
(\mathrm{n}=35)\end{array}$ & $\begin{array}{l}300 \mathrm{mg} \\
(\mathrm{n}=37)\end{array}$ & $\begin{array}{l}600 \mathrm{mg} \\
(\mathrm{n}=28)\end{array}$ & & \\
\hline & \multicolumn{4}{|c|}{$\mathrm{N}$, subjects $(\%)$ per treatment arm } & & \\
\hline \multicolumn{7}{|l|}{ Clinical outcome } \\
\hline 6 month relapse-free $(n=126)$ & $18(53)$ & $17(49)$ & $32(86)$ & $26(93)$ & $23(74)$ & $10(29)$ \\
\hline Relapsed $(n=73)$ & $16(47)$ & $18(51)$ & $5(14)$ & $2(7)$ & $8(26)$ & $24(71)$ \\
\hline \multicolumn{7}{|l|}{ Country } \\
\hline India $(\mathrm{n}=3)$ & $1(3)$ & $1(3)$ & $1(3)$ & $0(0)$ & $0(0)$ & $0(0)$ \\
\hline Peru $(n=114)$ & $20(59)$ & $21(60)$ & $19(51)$ & $16(57)$ & $18(58)$ & $20(59)$ \\
\hline Thailand $(\mathrm{n}=82)$ & $13(38)$ & $13(37)$ & $17(46)$ & $12(43)$ & $13(42)$ & $14(41)$ \\
\hline
\end{tabular}


Table 2 Distribution of CYP2D6 genotype, metabolizer phenotype and activity score

\begin{tabular}{|c|c|c|c|c|c|c|}
\hline $\begin{array}{l}\text { CYP2D6 } \\
\text { genotype }\end{array}$ & $\begin{array}{l}\text { CYP2D6 metabo- } \\
\text { lizer phenotype }\end{array}$ & $\begin{array}{l}\text { CYP2D6 activity } \\
\text { score }^{b}\end{array}$ & $\begin{array}{l}\text { All subjects } \\
n=199 \mathrm{~N}(\%)\end{array}$ & India $\mathrm{n}=3 \mathrm{~N}(\%)$ & $\begin{array}{l}\text { Peru } n=114 \mathrm{~N} \\
(\%)\end{array}$ & $\begin{array}{l}\text { Thailand } \mathrm{n}=82 \\
\mathrm{~N}(\%)\end{array}$ \\
\hline$* 4 / * 4$ & PM & 0 & $1(0.5)$ & - & $1(0.9)$ & - \\
\hline$* 4 / * 41$ & $\mathrm{IM}$ & 0.5 & $1(0.5)$ & - & $1(0.9)$ & - \\
\hline$* 1 / * 4$ & IM & 1.0 & $11(5.5)$ & - & $8(7.0)$ & $3(3.7)$ \\
\hline${ }^{*} 10 /{ }^{*} 10$ & IM & 1.0 & $19(9.5)$ & - & - & $19(23.2)$ \\
\hline${ }^{*} 10 / * 41$ & IM & 1.0 & $2(1)$ & - & - & $2(2.4)$ \\
\hline${ }^{*} 2 /{ }^{*} 4$ & IM & 1.0 & $3(1.5)$ & - & $3(2.6)$ & - \\
\hline$* 41 / * 41$ & $\mathrm{IM}$ & 1.0 & $2(1)$ & - & - & $2(2.4)$ \\
\hline${ }^{*} 1 /{ }^{*} 10$ & EM & 1.5 & $15(7.5)$ & - & - & 15 (18.3) \\
\hline$* 1 / * 41$ & EM & 1.5 & $6(3)$ & - & $3(2.6)$ & $3(3.7)$ \\
\hline *1/*9 & EM & 1.5 & $2(1)$ & - & $2(1.8)$ & - \\
\hline$*^{*} 2 /{ }^{*} 10$ & EM & 1.5 & $14(7)$ & - & - & $14(17.1)$ \\
\hline$* 2 /{ }^{*} 41$ & EM & 1.5 & $4(2)$ & - & - & $4(4.9)$ \\
\hline$*^{*} 1 /{ }^{*} 1$ & EM & 2.0 & 47 (23.6) & - & 35 (30.7) & $12(14.6)$ \\
\hline${ }^{*} 1 /{ }^{*} 2$ & EM & 2.0 & 45 (22.6) & $3(100)$ & $40(35.1)$ & $2(2.4)$ \\
\hline${ }^{*} 2 /{ }^{*} 2$ & EM & 2.0 & 27 (13.6) & - & $21(18.4)$ & $6(7.3)$ \\
\hline
\end{tabular}

a CYP2D6 poor metabolizers (PM), intermediate metabolizers (IM), extensive metabolizers (EM)

${ }^{b}$ CYP2D6 activity score is the sum of the per-allele scores; a null allele having a score of 0 , a deficient allele a score of 0.5 and a normal allele a score of 1 [12]

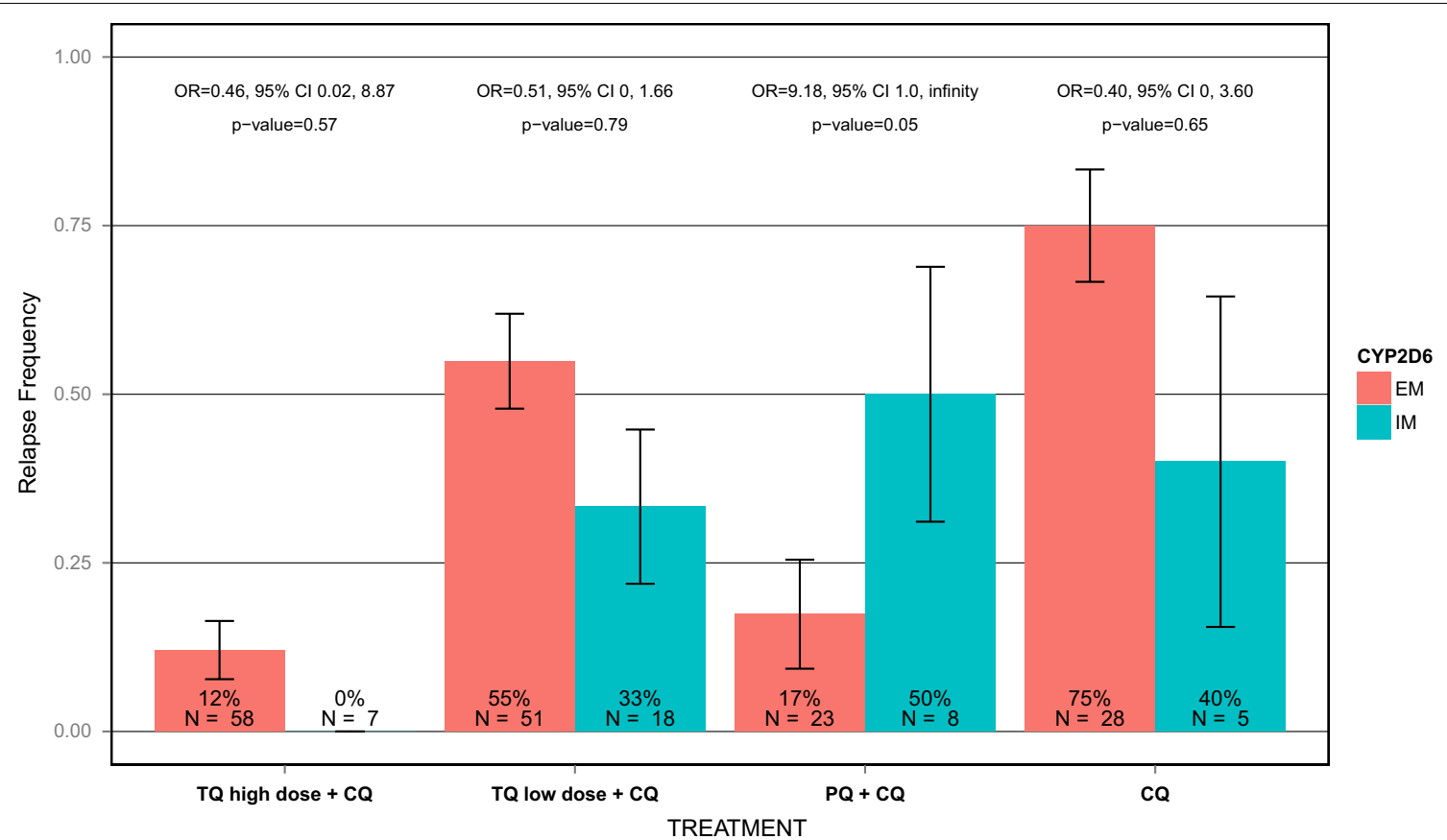

Fig. 1 Clinical outcome by treatment and CYP2D6 status. Relapse frequency is the percentage (\%) of subjects who experienced a relapse, with error bars representing the standard error of the mean. Odds ratios (ORs) and $95 \%$ confidence intervals ( $95 \% \mathrm{Cl}$ ) were from analyses comparing the relapse frequency of reduced metabolizers (IMs) with that of extensive metabolizers (EMs) within each treatment group. Exact logistic regression was used to derive ORs, except for the TQ high dose arm (TQ 300 mg/TQ 600 mg), in which Fisher's Exact test was used with a correction of 0.05 to each cell as there were no IM subjects who relapsed. One-sided p values ( $p$ ) are displayed (see "Methods" section) 
No evidence of association between CYP2D6 IM phenotype and increased frequency of clinical relapse of $P$. vivax infection was seen in either of the TQ treatment groups using a one-sided test as described in the Methods (Fig. 1). Relapse frequencies were actually lower in IM subjects in both TQ treatment groups, a trend that was also observed in the CQ only arm. Notably, all seven IM subjects in the TQ high dose $(300 / 600 \mathrm{mg})$ treatment group remained relapse-free during the 6 months of follow-up. By contrast, and in support of previous findings $[5,7]$, the relapse frequency was higher in PQtreated subjects who were IM (50\%) than in EM subjects $(17 \%), p=0.05$, odds ratio $=9.18(95 \%$ CI $1.00, \infty)$. The single PM subject in the clinical study was randomized to the CQ arm and relapsed. Comparable results were obtained using the CYP2D6 AS approach: no effect was observed in the TQ arms or in the CQ arm, whether the PM subject was included in the CQ arm or not, while a non-significant trend for lower AS scores in subjects who relapsed was observed in the PQ arm $(\mathrm{p}=0.06)$.
CYP2D6 phenotype had no significant effect on TQ AUC in either the TQ high dose $(\mathrm{p}=0.72)$ or TQ low dose $(\mathrm{p}=0.24)$ groups (Fig. 2$)$. The median AUC (interquartile range) in the TQ high dose group was 125.26 $\mu \mathrm{g} . \mathrm{h} / \mathrm{ml}(100.34-179.65 \mu \mathrm{g} . \mathrm{h} / \mathrm{ml})$ in EM subjects and $119.52 \mu \mathrm{g} . \mathrm{h} / \mathrm{ml}(93.17-136.67 \mu \mathrm{g} . \mathrm{h} / \mathrm{ml})$ in IM subjects. In the TQ low dose group, the median AUC (interquartile range) was $24.88 \mu \mathrm{g} . \mathrm{h} / \mathrm{ml}(17.86-36.50 \mu \mathrm{g} . \mathrm{h} / \mathrm{ml})$ in EM subjects and $34.92 \mu \mathrm{g} . \mathrm{h} / \mathrm{ml}(16.89-37.04 \mu \mathrm{g} . \mathrm{h} / \mathrm{ml})$ in IM subjects.

In vitro metabolism of TQ studied in CYP Supersomes $^{\text {TM }}$ and human hepatocytes indicated minimal metabolism of TQ. A summary of the structural information for the drug-related components of TQ are shown in Fig. 3, however, it should be noted that the majority of these components were observed in both sample and control incubations at comparable levels and are therefore likely to be due to degradation of TQ, and not true metabolites. In all incubations (including controls), the prominent component was TQ, with

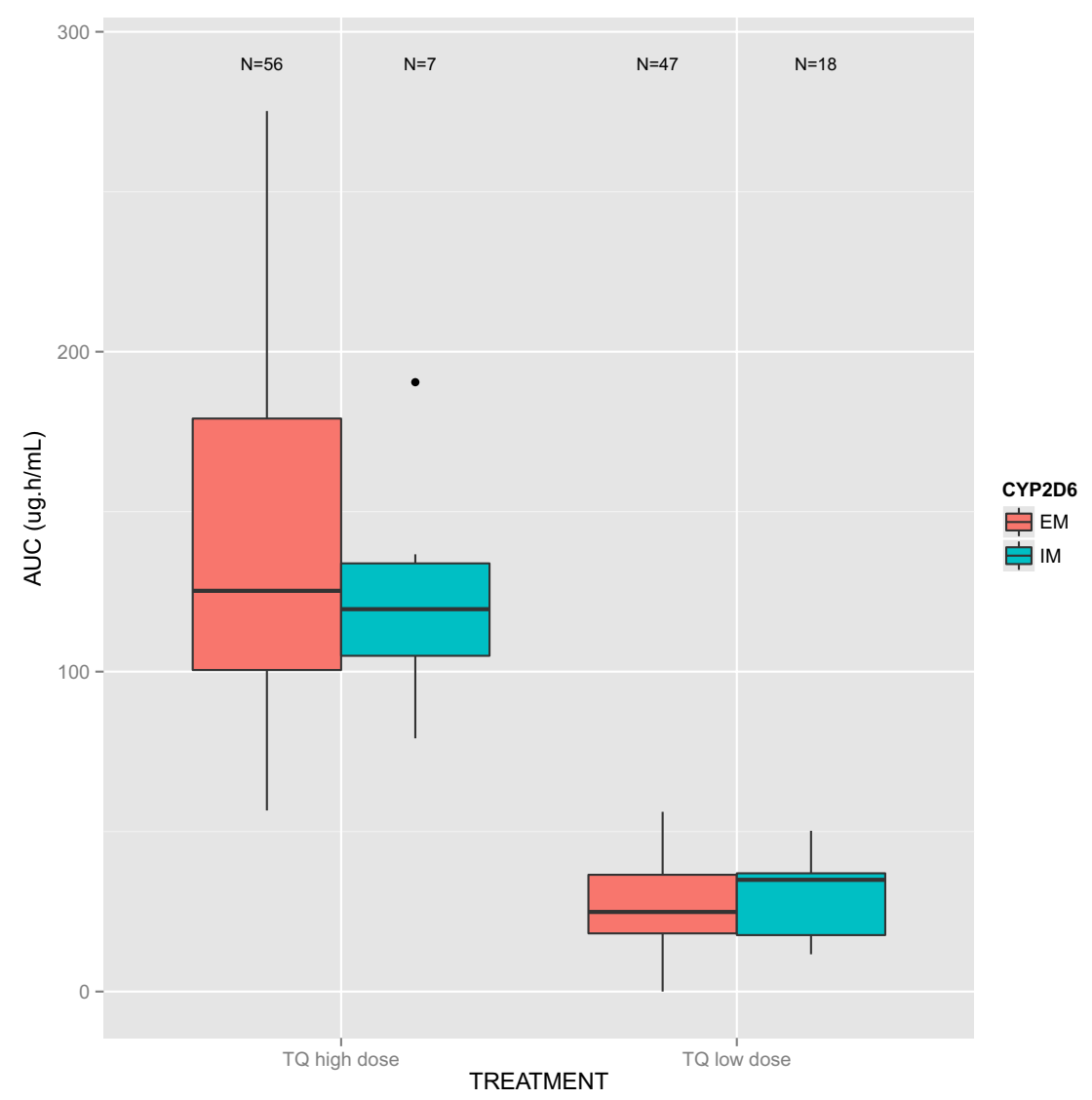

Fig. 2 Box whisker plot of TQ AUC by CYP2D6 status. The box whisker plots are displayed separately for the TQ high dose arm (TQ $300 \mathrm{mg} / \mathrm{TQ} 600 \mathrm{mg}$ ) and TQ low dose (TQ $50 \mathrm{mg} / \mathrm{TQ} 100 \mathrm{mg}$ ) arms, respectively. The median AUC is represented by the thick horizontal line, while the lower and upper box edges ('hinges') represent the quartiles (the 25th and 75th percentiles). The interquartile range (IQR) is the distance between the first and third quartiles. The upper (lower) whisker extends from the upper (lower) hinge to the highest (lowest) value that is within $1.5 *$ IQR of the hinge 


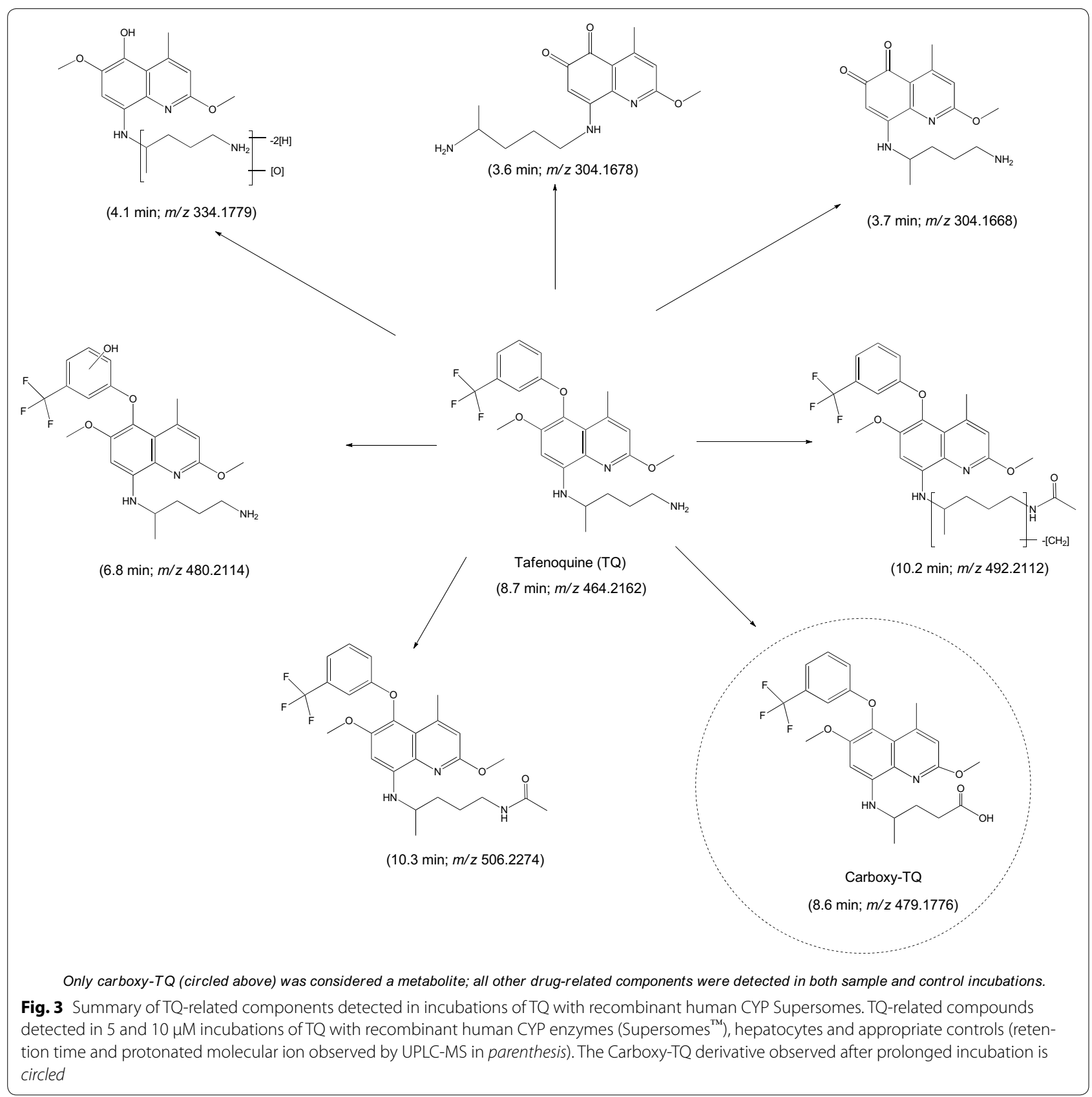

additional components resulting from $\mathrm{O}$-demethylation, $\mathrm{O}$-dearylation, oxidation, and $\mathrm{N}$-acetylation. Following 120 -min incubations of 5 and $10 \mu \mathrm{M}$ TQ with Supersomes $^{\mathrm{TM}}$ containing overexpressed CYP1A2, CYP2C8, CYP2C9, CYP2C19, CYP2D6, or CYP3A4, no additional drug-related components were detected which were not observed in the control incubations, indicating no or negligible metabolism of TQ had occurred. Following a six-day human hepatocyte relay, a minor component, carboxy-TQ, was the only additional TQ-related component detected by UPLC-MS. Carboxy-TQ was not observed in control incubations, indicating that minimal metabolism had occurred even after this long incubation period and despite a good metabolic capacity of the preparation (viability of the hepatocytes used ranged from 91-93\%).

\section{Discussion}

PQ has been approved for treating relapsing malaria since its discovery in the 1950s [16], however, it is not widely 
used in areas where $P$. vivax is endemic [1]. Factors that have hampered PQ's effectiveness in developing countries include poor compliance, due to a lengthy treatment regime (typically 14 days), and concerns around the risk of haemolysis in G6PD-deficient individuals. While both PQ and TQ have G6PD liability, TQ's long half-life has enabled a single dose regime in current clinical trials, which could be expected to result in good patient compliance.

As highlighted recently, a potential CYP2D6 PGx liability for 8-AQs would represent a significant obstacle to large-scale prophylaxis and eradication campaigns for P. vivax [8]. Here, a retrospective PGx analysis was performed on the effect of inferred CYP2D6 phenotype on TQ PK and TQ relapse efficacy in participants from TAF112582, a published randomized phase $2 \mathrm{~b}$ clinical trial [2]. Given the known global variation in allele frequencies, the difference in CYP2D6 metabolizer phenotype observed between subjects from Thailand and Peru was as expected and driven primarily by CYP2D6*10, a functionally-deficient allele common in Asian countries [17]. These results demonstrate that TQ efficacy is not diminished in $P$. vivax-infected individuals who have reduced CYP2D6 metabolism nor is TQ PK affected by CYP2D6, a finding that is at odds with previously published research in mice $[8,9]$. However, these data support the growing body of evidence implicating reduced CYP2D6 metabolism in PQ failure [3-7].

In contrast to $\mathrm{PQ}$ [4], metabolites of $\mathrm{TQ}$, in particular those potentially resulting from CYP2D6 metabolism, are considered less likely to influence the efficacy of this drug, which agrees with findings from the PGx and PK analyses conducted in this report. At present, the mechanism of action for TQ is not known as few metabolites have been observed in subjects dosed with TQ. Further support for a differential effect of CYP2D6 on TQ and PQ metabolism comes from various in vitro human liver drug metabolism investigations (including those described within this publication), all of which have demonstrated minimal hepatic metabolic turnover of TQ. This would suggest that unlike $P Q$, metabolism of $T Q$ may not be an important factor in vivo for efficacy against $P$. vivax. Unpublished analyses for TQ metabolites in plasma (and blood) collected from healthy female volunteers dosed orally with TQ (up to $300 \mathrm{mg}$ ) for 3 days are consistent with in vitro findings reported here. The only circulating oxidative metabolite of TQ observed in 0-144-hour pools was carboxy-TQ, which was estimated by ultraviolet spectroscopy and mass spectrometry to be at very low levels compared to unchanged TQ (unpublished data on file within DMPK Department, GSK).

Taken together, the in vivo and in vitro data presented here provide evidence that TQ efficacy against $P$. vivax might not be reliant on CYP2D6 metabolism to the same extent as PQ. As indicated previously, the translation of genotype information into metabolizer phenotype is challenging and small sample size limits conclusions that can be drawn from the PGx component of this study, including whether TQ efficacy is different in CYP2D6 $\mathrm{PM}$ and IMs. Therefore, the current hypothesis and any potential CYP2D6 effects on TQ and PQ efficacy will be further evaluated in the ongoing phase 3 programmes (TAF112582 Part 2 and TAF116564).

\section{Conclusion}

Converging evidence suggests that TQ $P$. vivax radical cure efficacy in humans is not impacted in individuals with decreased CYP2D6 metabolism to the same extent as $\mathrm{PQ}$.

\section{Additional files}

Additional file 1. The percentage of intermediate metabolizers (IM) by country. The percentage of subjects who were intermediate metabolizers (IM) is shown with error bars representing the standard error of the mean. The three subjects from India are not shown as all were extensive metabolizers (EMs).

Additional file 2. The percentage of intermediate metabolizers by treatment. The percentage of subjects who were intermediate metabolizers (IM) with error bars representing the standard error of the mean.

\section{Abbreviations}

8-AQ: 8-aminoquinoline; AS: CYP2D6 activity score; AUC: area under the curve; CQ: chloroquine; CYP: cytochrome P450; DMPK: drug metabolism and pharmacokinetics; EM: extensive metabolizer; FDA: federal drug administration; G6P: glucose-6-phosphate; G6PD: glucose-6-phosphate dehydrogenase; GSK: GlaxoSmithKline; HPLC: high-performance liquid chromatography; IM: intermediate metabolizer; ITT: intent to treat; MMV: medicines for malaria venture; NADP: $\beta$-nicotinamide adenine dinucleotide phosphate; PGX: pharmacogenetics; PM: poor metabolizer; PQ: primaquine; TQ: tafenoquine; UPLC-MS: ultra performance liquid chromatography-mass spectrometry; w/v: weight per volume.

\section{Authors' contributions}

ALC, RR and SK provided the study samples; PS, JG, JR, ZX, MT, SD, and NC conceived and designed the study; PS, MT, CB, and ZX analysed the data; PS, $J G, J R, Z X, S D$, and MT prepared the first draft of the manuscript; All authors read and approved the final manuscript.

\section{Author details}

1 PAREXEL International, 2520 Meridian Parkway, Durham, NC 27713, USA. ${ }^{2}$ GlaxoSmithKline, Stockley Park West, Uxbridge, Middlesex, UK. ${ }^{3}$ Medicines for Malaria Venture, Geneva, Switzerland. ${ }^{4}$ GlaxoSmithKline, Ware, Hertfordshire, UK. ${ }^{5}$ Instituto de Medicina Tropical, Alexander von Humboldt, Universidad Peruana Cayetano Heredia, Lima, Peru. ${ }^{6}$ Mae Sot Hospital, Mae Sot, Tak Province, Thailand. ${ }^{7}$ Faculty of Tropical Medicine, Mahidol University, Bangkok, Thailand. ${ }^{8}$ Department of Pathology, University of Melbourne, Melbourne, VIC, Australia.

\section{Acknowledgements}

We thank Navin Goyal who advised on the TAF112582 TQ pharmacokinetic data analysis.

Pamela L. St Jean, Zhengyu Xue and Justin P. Rubio Previously employed by GlaxoSmithKline. Work performed on this publication was carried out while employed by GlaxoSmithKline, Research Triangle Park, NC, USA and Stevenage, Hertfordshire, UK. 


\section{Competing interests}

GK, JG, NC, MT, and CB are full time employees of, and hold stock in, GSK; PS, ZX and JR were full-time employees of GSK during the time of this study and currently hold stock in GSK; SD is a full-time employee of MMV; AL-C, RR and SK declare no competing interests.

Received: 3 December 2015 Accepted: 4 February 2016

Published online: 18 February 2016

\section{References}

1. Price RN, Tjitra E, Guerra CA, Yeung S, White NJ, Anstey NM. Vivax malaria: neglected and not benign. Am J Trop Med Hyg. 2007;77(6 Suppl):79-87.

2. Llanos-Cuentas A, Lacerda MV, Rueangweerayut R, Krudsood S, Gupta SK, Kochar SK, et al. Tafenoquine plus chloroquine for the treatment and relapse prevention of Plasmodium vivax malaria (DETECTIVE): a multicentre, double-blind, randomised, phase $2 \mathrm{~b}$ dose-selection study. Lancet. 2014:383:1049-58.

3. Pybus BS, Sousa JC, Jin X, Ferguson JA, Christian RE, Barnhart R, et al. CYP450 phenotyping and accurate mass identification of metabolites of the 8-aminoquinoline, anti-malarial drug primaquine. Malar J. 2012;11:259.

4. Pybus BS, Marcsisin SR, Jin X, Deye G, Sousa JC, Li Q, et al. The metabolism of primaquine to its active metabolite is dependent on CYP 2D6. Malar J. 2013:12:212.

5. Bennett JW, Pybus BS, Yadava A, Tosh D, Sousa JC. Primaquine failure and cytochrome P-450 2D6 in Plasmodium vivax malaria. N Engl J Med. 2013;369:1381-2

6. Potter BM, Xie LH, Vuong CT, Zhang J, Zhang P, Duan D, et al. Differential CYP 2D6 metabolism alters primaquine pharmacokinetics. Antimicrob Agents Chemother. 2015;59:2380-7.

7. Ingram RJH, Crenna-Darusallam C, Soebianto S, Noviyanti R, Baird JK. The clinical and public health problem of relapse despite primaquine therapy: case review of repeated relapses of Plasmodium vivax acquired in Papua New Guinea. Malar J. 2014;13:488.
8. Marcsisin SR, Sousa JC, Reichard GA, Caridha D, Zeng Q, Roncal N, et al. Tafenoquine and NPC-1161B require CYP 2D metabolism for anti-malarial activity: implications for the 8-aminoquinoline class of anti-malarial compounds. Malar J. 2014;13:2.

9. Vuong C, Xie LH, Potter BMJ, Zhang J, Zhang P, Duan D, et al. Differential CYP 2D6 metabolism alters tafenoquine pharmacokinetics. Antimicrob Agents Chemother. 2015;59:3864-9.

10. Zhou SF. Polymorphism of human cytochrome P450 $2 D 6$ and its clinical significance: Part I. Clin Pharmacokinet. 2009:48:689-723.

11. Zhou SF. Polymorphism of human cytochrome P450 $2 \mathrm{D} 6$ and its clinical significance: Part II. Clin Pharmacokinet. 2009:48:761-804.

12. Gaedigk A, Simon SD, Pearce RE, Bradford LD, Kennedy MJ, Leeder JS. The CYP2D6 activity score: translating genotype information into a quantitative measure of phenotype. Clin Pharmacol Ther. 2008;83:234-42.

13. Rebsamen MC, Desmeules J, Daali Y, Chiappe A, Diemand A, Rey C, et al. The Amplichip CYP450 test: cytochrome P450 2D6 genotype assessment and phenotype prediction. Pharmacogenomics J. 2009;9:34-41.

14. Tenero D, Green JA, Goyal N. Exposure-response analyses for tafenoquine after administration to patients with Plasmodium vivax malaria. Antimicrob Agents Chemother. 2015;59:6188-94.

15. Di L, Trapa P, Obach RS, Atkinson K, Bi YA, Wolford AC, et al. A novel relay method for determining low-clearance values. Drug Metab Dispos. 2012;40:1860-5.

16. Edgcomb JH, Arnold J, Yount EH Jr, Alving AS, Eichelberger L, Jeffery GM, et al. Primaquine, SN 13272, a new curative agent in vivax malaria; a preliminary report. J Natl Malar Soc. 1950;9:285-92.

17. Sistonen J, Sajantila A, Lao O, Corander J, Barbujani G, Fuselli S. CYP2D6 worldwide genetic variation shows high frequency of altered activity variants and no continental structure. Pharmacogenet Genomics. 2007;17:93-101.

\section{Submit your next manuscript to BioMed Central and we will help you at every step:}

- We accept pre-submission inquiries

- Our selector tool helps you to find the most relevant journal

- We provide round the clock customer support

- Convenient online submission

- Thorough peer review

- Inclusion in PubMed and all major indexing services

- Maximum visibility for your research

Submit your manuscript at www.biomedcentral.com/submit

\section{(O) Biomed Central}

